\title{
A PROSPECTIVE STUDY OF ADVERSE DRUG REACTIONS DUE TO PLATINUM ANALOGS - CHEMOTHERAPY IN A TERTIARY CARE HOSPITAL
}

\author{
SUMIT KUMAR ${ }^{1}$, BADRUDDEEN ${ }^{2 *}$, SINGH S P ${ }^{1}$, MOHAMMAD IRFAN KHAN ${ }^{2}$ \\ ${ }^{1}$ Department of Pharmacology and Therapeutics, GSVM Medical College, Kanpur, Uttar Pradesh, India. ${ }^{2}$ Department of Pharmacy, Faculty \\ of Pharmacy, Integral University Lucknow, Uttar Pradesh, India. Email: badarmiracle@gmail.com
}

Received: 19 January 2018, Revised and Accepted: 01 March 2018

\section{ABSTRACT}

Objective: The objective of this study was to analyze the types of adverse drug reactions (ADRs) associated with platinum analogs (cisplatin, carboplatin and oxaliplatin) used for cancer chemotherapy in a tertiary care hospital and determine their causal relationship with the offending drug.

Methods: This prospective, observational, non-interventional study was conducted in a tertiary care hospital at GSVM Medical College Kanpur, India, for 4 months. Patients of all age and either sex were included in the study. ADRs were reported by the physicians of oncology department of the hospital and ADRs were assessed for different parameters -causality, outcome, and seriousness of ADR as per the World Health Organization (WHO), type of ADRs as per expanded Rawlins and Thompson's classification, predictability using council for international organization of medical sciences guidelines and severity using modified Hartwig's scale. Descriptive statistics were used for data analysis.

Results: A total of 140 ADRs were reported from platinum analogs following treatment of different types of cancer in hospital. The burden of ADRs in each patient was 2.41. Most of the ADRs were observed in the age group of 40-60 years. Vomiting (27 ADRs) was commonly reported reaction. Among platinum analogs, cisplatin leads to 82 ADRs (58.57\%) followed by carboplatin with 53 ADRs (37.86\%) and least with oxaliplatin 5 ADRs (3.57\%). Most of the ADRs on causality assessment were possible $(104,74.29 \%)$ and probable $(36,25.71 \%)$ in nature. Type $-A$ ADRs account for $4 / 5^{\text {th }}$ of the total reported ADRs, followed by Type-B and C. Severity of $90.71 \%$ ADRs was found to be mild followed by moderate, with no case of severe and serious nature. Nearly, most of the ADRs were of predictable type (97.14\%).

Conclusion: The potential of platinum analogs to cause ADRs is high; thus, the need of effective ADRs monitoring is highly emphasized.

Keywords: Adverse drug reactions, Chemotherapy, Causality, Platinum analogs.

(C) 2018 The Authors. Published by Innovare Academic Sciences Pvt Ltd. This is an open access article under the CC BY license (http://creativecommons. org/licenses/by/4. 0/) DOI: http://dx.doi.org/10.22159/ajpcr.2018.v11i6.24849

\section{INTRODUCTION}

An adverse drug reaction (ADR) is any noxious, unintended, and undesired effect of a drug that occurs at doses used for prophylaxis, diagnosis or therapy or for modification of physiological function $[1,2]$. The prevalence of ADRs of anticancer drugs in Indian context is 10$12 \%$ [3]. Cancer is one of the leading causes of death worldwide with estimated $12 \%$ deaths annually [4]. Platinum analogs have become the mainstay of treatment for many tumors including ovarian cancer, lung cancer, germ cell tumors, head and neck cancer, bladder cancer and to a lesser degree breast cancer and gastric cancer [5].

Platinum analogs-antineoplastic drugs are coordination complexes of platinum, cause crosslinking of DNA as monoadduct, interstrand crosslinks, intrastrand crosslinks, or DNA protein crosslinks. Mostly they act on the adjacent N-7 position of guanine, forming a 1, 2 intrastrand crosslink, this crosslinking inhibits DNA repair and/or DNA synthesis in cancer cells. Main approved platinum-based anticancer drugs include cisplatin, carboplatin, oxaliplatin and nedaplatin $[6,7]$.

Cisplatin has major antitumor activity in a broad range of solid tumors including non-small cell and small cell lung cancer, esophageal and gastric cancer, head and neck cancer and genitourinary cancers, particularly testicular, ovarian, and bladder cancer. Cisplatin is a highly emetic drug. Antiemetics are routinely administered before infusing it. The most important toxicity is renal impairment which is dependent on total dose administered and can be reduced by maintaining good hydration. Tinnitus, deafness, sensory neuropathy and hyperuricemia are other problems.
Carboplatin is a second-generation platinum analogs, better tolerated and has a toxicity profile different from cisplatin, but mechanism of action and clinical utility are similar. Nephrotoxicity, ototoxicity and neurotoxicity are low. Nausea and vomiting is milder and is delayed. The dose-limiting toxicity is thrombocytopenia and less often leucopenia. Intravenous hydration is not required, and for this reason, carboplatin has now widely replaced cisplatin in combination chemotherapy.

Oxaliplatin is a third-generation platinum analog. Its mechanism of action is identical to that of cisplatin and carboplatin. However, cancer cells that are resistant to cisplatin or carboplatin on the basis of mismatch repair defects are not cross-resistant to oxaliplatin. Neurotoxicity is dose limiting and characterized by a peripheral sensory neuropathy. Myelosuppression is modest, but diarrhea and acute allergic reactions are reported $[8,9]$.

Cancer is one of the leading causes of mortality around globe. As these drugs have narrow therapeutic window, hence chances of ADRs with anticancer drugs are higher and in addition, hospital stay also significantly increases that ultimately influence the treatment cost and quality of life. The estimated total cost of treatment for ADRs is $1.7 \%$ of the total hospital budget with a median cost of 8517 francs [10]. During chemotherapy, most of the ADRs go unreported due to unknowingness of health-care professionals, lack of time to report and insufficient staff in the hospitals.

Hence, it becomes crucial to agnise the pattern of ADRs occurring with most commonly used platinum analogs-anticancer drugs so as to heighten the quality of life and to reduce the treatment cost of ADRs 
associated hospitalization among cancer patient receiving platinum analogs singly or in combination with other drugs.

\section{METHODS}

A prospective, observational, non-interventional study carried out at in-patient and out-patient setting in the Oncology Department of GSVM Medical College, Kanpur, U.P, for a period of 4 months (January 2017-April 2017). The patients received cancer chemotherapy as per the assessment of the treating physician. No changes in the treatment decision, schedule or duration were made during the study. Permission from the institutional ethical committee was obtained before the initiation of this study.

\section{Inclusion criteria's}

- $\quad$ Patients of all age groups and either sex.

- All the suspected ADRs that may be due to the platinum analogs as single drug regimen or in combination of two or more drugs.

\section{Exclusion criteria's}

- Patients who were mentally retarded and unconscious

- Patients who consumed alternative system of medicine.

- Adverse events caused by administration errors, noncompliance or overdose.

Patient demographic data such as patient initial, OPD/IPD number, age, sex, medical history, medication history, surgery history, allergies, management and outcome of ADR had been recorded on the suspected ADRs reporting form of Indian Pharmacopoeia Commission. ADRs were evaluated using different scales as follows:

1. Causality of ADRs - World Health Organization (WHO)-UMC assessment scale.

2. Outcome and seriousness of ADR - WHO

3. Type of ADR - expanded Rawlins and Thompson's classification.

4. Severity of ADRs - modified Hartwig's scale.

5. Predictability of ADRs - council for international organization of medical sciences (CIOMS).

ADRs were further evaluated to determine most common drug, organ system, and reaction associated with platinum analogs and descriptive statistics were used for data analysis.

\section{RESULTS}

In the presentstudy, the most common age group that developed maximum number of ADRs is 40-60 years followed by age group 20-40 years and in female population more number of ADRs (66.43\%) had appeared followed by male patients (33.57\%) as shown in Table 1 and Fig. 1 . Cisplatin was the drug that leads to maximum ADRs (58.57\%) followed by carboplatin (37.86\%) and least ADRs were reported with oxaliplatin (3.57\%) as shown in Fig. 2.

The most common ADR with platinum analogs was vomiting (27) followed with alopecia, diarrhea, anorexia, black pigmentation of nail, constipation, decrease in hemoglobin, nausea, nervousness, headache,

Table 1: Distribution of ADRs of platinum analogs-chemotherapeutic agent as per patient age and gender

\begin{tabular}{lll}
\hline Particulars & $\begin{array}{l}\text { Number of patient } \\
\mathbf{n = 5 8}(\mathbf{6})\end{array}$ & $\begin{array}{l}\text { Number of ADRs } \\
\mathbf{n = 1 4 0}(\%)\end{array}$ \\
\hline $\begin{array}{l}\text { Gender } \\
\quad \text { Male }\end{array}$ & $22(38)$ & $47(33.57)$ \\
$\quad$ Female & $36(62)$ & $93(66.43)$ \\
Age group (years) & & \\
$0-20$ & 0 & 0 \\
$20-40$ & $12(20.68)$ & $26(18.57)$ \\
$40-60$ & $37(63.80)$ & $95(67.86)$ \\
$>60$ & $9(15.52)$ & $19(13.57)$ \\
\hline
\end{tabular}

ADRs: Adverse drug reactions dizziness and mucositis as shown in Table 2 and Fig. 3. ADRs mostly involved with digestive system include $51 \%$ cases, followed by dermatological system in $24 \%$ cases, $10 \%$ cases of ADRs were related to nervous system, $6 \%$ cases involved cardiovascular system and in $5 \%$ cases, musculoskeletal system was involved as shown in Fig. 4.

Using different parametric scale, ADRs were evaluated for the type of ADRs as per expanded Rawlins and Thompson's classification most of ADRs were of Type-A (78.57\%) followed by Type-B (14.29\%) and Type-C, whereas no case of Types-D-F ADRs. On modified Hartwig's scale, most of ADRs were of mild nature (90.71\%) followed by moderate (9.29\%), no case of severe category. As per the WHO seriousness criteria, no serious case was reported with platinum analogs and in predictability assessment as per CIOMS $97.14 \%$ cases of ADRs were of predictable type and rest $2.86 \%$ ADRs comes under category of not predictable. As per the WHO causality assessment, $74.29 \%$ ADRs are of possible type followed with $25.71 \%$ probable. The outcome of maximum ADRs was found to be recovered $-66.42 \%$ followed with $26.43 \%$ not recovered, $6.43 \%$ ADRs were found to be recovering and outcome of $0.72 \%$ ADRs was unknown as shown in Table 3.

\section{DISCUSSION}

Platinum analogs are the most commonly used anticancer drugs and well associated with ADRs such as nausea, vomiting, renal toxicity, peripheral neuropathy, hypersensitivity reactions, electrolyte disturbances, ototoxicity, and myelosuppression [11]. Even though an adequate premedication with dexamethasone, ranitidine, and ondansetron was given to each

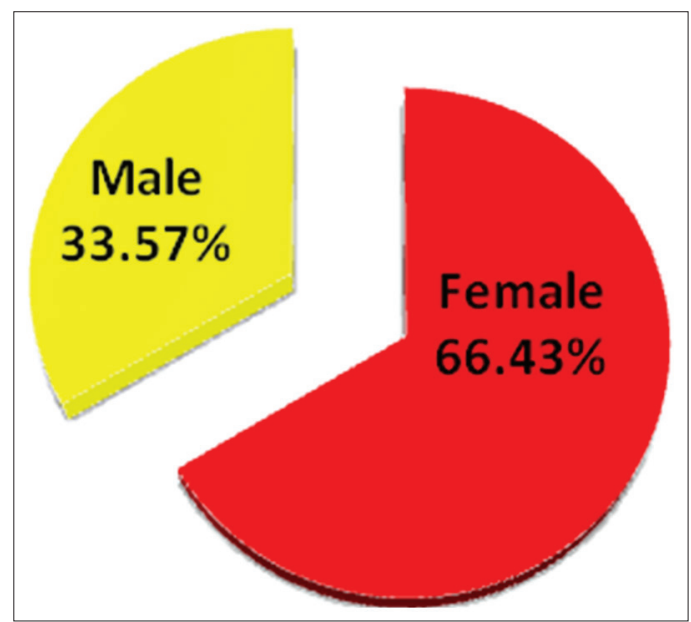

Fig. 1: Distribution of adverse drug reactions in male and female population

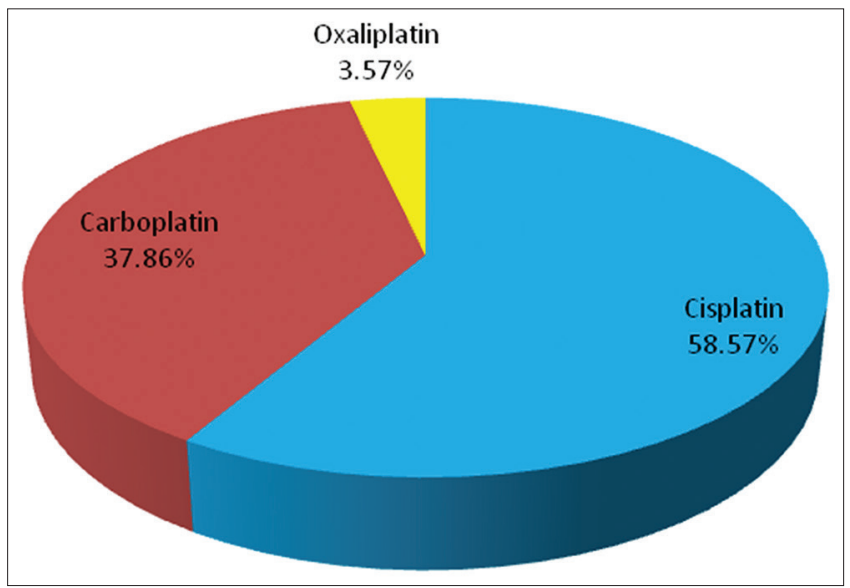

Fig. 2: Percentage share of drugs in causing adverse drug reactions 


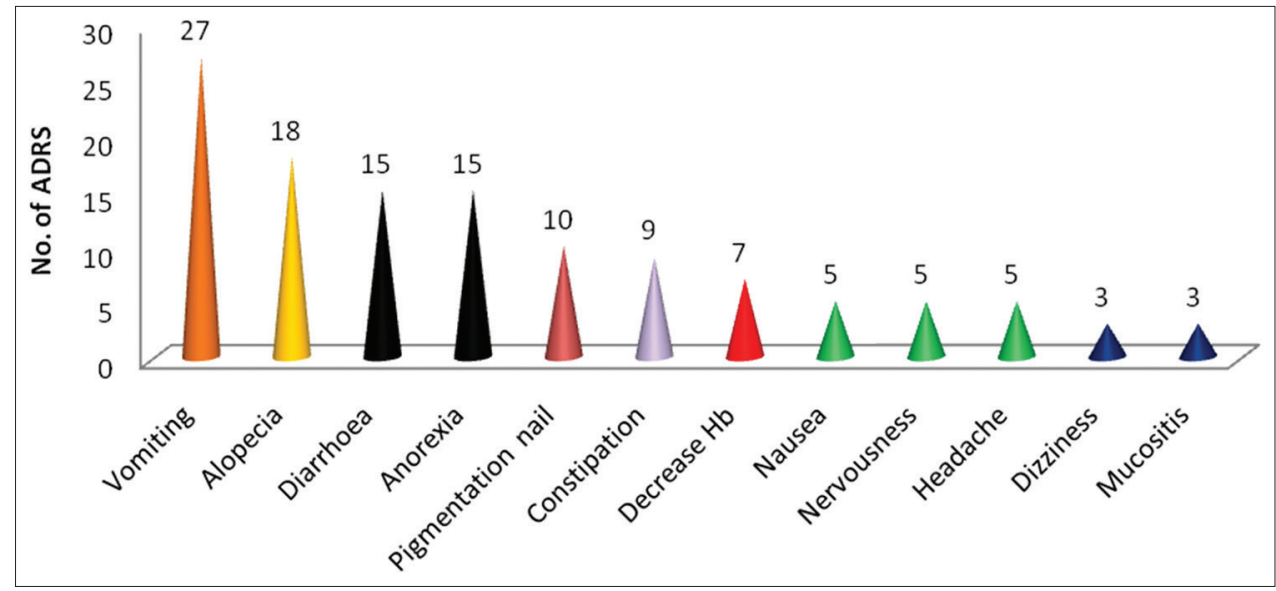

Fig. 3: Most common types of reaction associated with drugs

Table 2: Various types of ADRs associated with platinum analogs

\begin{tabular}{ll}
\hline Types of reaction & Number of ADRs (\%) \\
\hline Alopecia & $18(12.85)$ \\
Anorexia & $15(10.71)$ \\
Body pain & $2(1.42)$ \\
Chest pain & $1(0.72)$ \\
Constipation & $9(6.42)$ \\
Decrease hemoglobin & $7(5)$ \\
Diarrhea & $15(10.71)$ \\
Dizziness & $3(2.14)$ \\
Dry mouth & $1(0.72)$ \\
Febrile neutropenia & $1(0.73)$ \\
Fever & $2(1.42)$ \\
Flatulence & $1(0.72)$ \\
Headache & $5(3.57)$ \\
Increased SGPT & $1(0.72)$ \\
Insomnia & $1(0.72)$ \\
Itching & $1(0.72)$ \\
Joint pain & $1(0.72)$ \\
Lethargy & $1(0.72)$ \\
Limb pain & $2(1.42)$ \\
Mucositis & $3(2.14)$ \\
Nausea & $5(3.57)$ \\
Nervousness & $5(3.57)$ \\
Numbness & $1(0.72)$ \\
Pigmentation of nail & $10(7.14)$ \\
Rashes & $1(0.72)$ \\
Tinnitus & $1(0.72)$ \\
Vomiting & $27(19.28)$ \\
\hline ADRs Adverse &
\end{tabular}

ADRs: Adverse drug reactions

patient, the frequency of vomiting remained high due to high emetogenic potential of these drugs. Neutropenia can be managed by sargramostim and filgrastim. The study focuses to avail adequate data of ADRs to improve the management of expected ADRs.

In our study, 140 ADRs are suspected with different platinum analogs, of which majority of ADRs were reported from female patients than from male. A study conducted by Swathi [12] and Chopra et al. [13] also showed female preponderance. This may be attributed to the smaller body surface area in females.

In the present study, the most suspected ADRs were vomiting (19.28\%) followed by alopecia (12.85\%), anorexia (10.71\%) and diarrhea $(10.71 \%)$, similarly the most common ADR in Swathi study was vomiting. Vomiting is very common side effects known with cancer chemotherapeutic agents [14].

Our study shows 40-60 age group patients having maximum number of ADRs. Similarly, a study of Behera etal. [15] showed ADRs in the age group 41-60 years

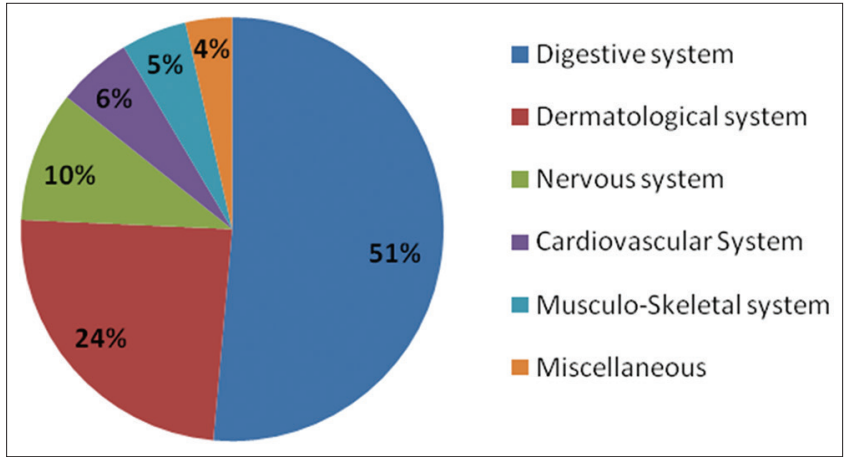

Fig. 4: Organ system involved with adverse drug reactions

was highest, similar result with a study of Sharma et al. [16]. This could be due to the higher incidence of cancers in this age group.

The most common offending platinum analog was cisplatin (58.57\%) followed by carboplatin $(37.86 \%)$ and least with oxaliplatin (3.57\%). It is quite similar to the study conducted by Swathi that showed the suspected drug was cisplatin (64.58\%) followed by carboplatin (25\%) and oxaliplatin $(10.41 \%)$; this may be due to more frequent use of cisplatin-based chemotherapy.

In our study, no serious ADRs were reported which were similar to the study of Swathi that also not reported any serious ADRs.

Most of the ADRs in our study were specified as possible (74.29.\%) followed by probable $(25.71 \%)$ in WHO-UMC causality assessment which is quite consistent with studies of Behera et al. (90.9\%) followed by probable, which was contrary to a study of Saini et al. [17] where most of the ADRs were having causality probable (64.67\%).

Our study shows most commonly involved organ system as digestive system (51\%), which was similar to the study conducted by Swathi with $70 \%$ cases of ADRs involving digestive system.

\section{CONCLUSION}

Platinum analogs have a high proclivity to cause ADRs owing to their action on fast-dividing cells. Hence, early detection of ADRs may be helpful in reducing the harm either by modifying the dose or substituting with suitable alternative and also plays major role in future for better prevention with similar ADRs, thereby patient's quality of life will improve and treatment cost burden will certainly reduce. In this study, causality of ADR was poor due to the presence of concomitant administered drugs. This study emphasizes the need of effective ADRs monitoring system in each and every hospital to reduce the burden of ADRs. 
Table 3: Evaluation of ADRs using different parametric scale

\begin{tabular}{ll}
\hline Particulars & Number of ADRs n=140 (\%) \\
\hline Type of ADRs & $110(78.57)$ \\
A & $20(14.29)$ \\
B & $10(7.14)$ \\
C & $127(90.71)$ \\
Severity & $13(9.29)$ \\
Mild & $0(0)$ \\
Moderate & $140(100)$ \\
Severe & $0(0)$ \\
Seriousness & $136(97.14)$ \\
Non-serious & $4(2.86)$ \\
Serious & \\
Predictability & $36(25.71)$ \\
Predictable & $104(74.29)$ \\
Not-predictable & \\
Causality & $93(66.42)$ \\
Probable & $9(6.43)$ \\
Possible & $37(26.43)$ \\
Outcome of reaction & $1(0.72)$ \\
Recovered & \\
Recovering & \\
Not recovered & Unknown
\end{tabular}

ADRs: Adverse drug reactions

\section{ACKNOWLEDGEMENT}

Authors are highly thankful to faculty of the Oncology Department, GSVM Medical College, Kanpur, and faculty of pharmacy Integral University Lucknow (IU/R\&D/2018-MCN000242) for providing suitable facilities and support for the successful completion of this study.

\section{CONFLICT OF INTEREST}

The authors declare no conflict of interest related with this manuscript.

\section{REFERENCES}

1. Parthasarathi G, Karin N, Milap CN. A Textbook of Clinical Pharmacy Practice, Essential Concepts and Skills. $2^{\text {nd }}$ ed. India: Universities Press Private Limited.; 2012.

2. Eby M, Chandrika C, Preethy MK, Srinivasa R. A prospective observational study on prescribing trends and adverse drug reactions in stroke patients. Int J Pharm Pharm Sci 2017;9:25-30.
3. Pirmohamed M, James S, Meakin S, Green C, Scott AK, Walley TJ. Adverse drug reactions as cause of admission to hospital: Prospective analysis of 18820 patients. B Med J 2004;329:15-9.

4. Mariotto AB, Yabroff KR, Shao Y, Feuer EJ, Brown ML. Projections of the cost of cancer care in the United States: 2010-2020. J Natl Cancer Inst 2011;103:117-28.

5. Sharwankumar S, Bhaswat C. Safety signal detection for platinum compounds in canadian spontaneous adverse event reports. Int J Pharm Pharm Sci 2017;6:405-11.

6. Poklar N, Pilch DS, Lippard SJ, Redding EA, Dunham SU, Breslauer KJ. Influence of cisplatin intrastrand crosslinking on the conformation, thermal stability, and energetics of a 20-mer DNA duplex. Proc Natl Acad Sci 1996;93:7606-11.

7. Rudd GN, Hartley JA, Souhami RL. Persistence of cisplatin-induced DNA interstrand crosslinking in peripheral blood mononuclear cells from elderly and young individual. Cancer Chemother Pharmacol 1995;35:323-6.

8. Katzung BG. Basic Clinical Pharmacology. $10^{\text {th }}$ ed. San Francisco: The McGraw-Hill Companies; 2007.

9. Tripathi KD. Essentials of Medical Pharmacology. $7^{\text {th }}$ ed. New delhi: Jaypee Brothers; 2013.

10. Couffignal AL, Lapeyre-Mestre M, Bonhomme C, Bugat R, Montastruc JL. Adverse effects of anticancer drugs: Apropos of a pharmacovigilance study at a specialized oncology institution. Therapie 2000;55:635-41.

11. Brunton LL, Chabner BA, Knollmann BC. Goodman \& Gilman's. The Pharmacological Basis of Therapeutics. $12^{\text {th }}$ ed. New York: McGraw Hill; 2011.

12. Swathi B. Adverse drug reaction profiles of commonly used platinum compounds in cancer chemotherapy. Int J Basic Clin Pharmacol 2015;4:284-9.

13. Chopra D, Harmeet SR, Sharma V, Mishra R. Chemotherapy-induced adverse drug reactions in oncology patients: A prospective observational survey. Indian J Med Paed Oncol 2016;37:42-6.

14. Jordan K, Sippel C, Schmoll HJ. Guidelines for antiemetic treatment of chemotherapy-induced nausea and vomiting: Past, present, and future recommendations. Oncology 2007;12:1143-50.

15. Behera SK, Kishtapati CR, Gunaseelan V, Dubashi B, Chandrasekaran A, Selvarajan S. Adverse drug reactions in cancer patient. Young Pharm 2017;9:593-97.

16. Sharma A, Kumari KM, Manohar HD, Bairy KL, Thoma SJ. Pattern of adverse drug reactions due to cancer chemotherapy in a tertiary care hospital in South India. Perspect Clin Res 2015;6:109-15.

17. Saini VK, Sewal RK, Ahmad Y, Medhi B. Prospective observational study of adverse drug reactions of anticancer drugs used in cancer treatment in a tertiary care hospital. Indian J Pharm Sci 2015;77:687-93. 